
$\$$ Research Square
Preprints are preliminary reports that have not undergone peer review.
They should not be considered conclusive, used to inform clinical practice, or referenced by the media as validated information.

\title{
Microscopic Transsphenoidal Resection of Pituitary Adenomas With Conchal Sphenoid Sinus: a Report of Three Cases
}

\section{Jianhe Zhang}

Department of Neurosurgery, The Affiliated Hospital of Putian University, Putian 351100, PR China Shousen Wang ( $D$ leo200303034@126.com )

Department of Neurosurgery, 900th Hospital, Fuzong Clinical College of Fujian Medical University, Fuzhou 350025, PR China

\section{Case report}

Keywords: conchal sphenoid sinus, non-pneumatized sphenoid sinuses, transsphenoidal, neuronavigation

Posted Date: October 30th, 2020

DOI: https://doi.org/10.21203/rs.3.rs-98903/v1

License: (c) (i) This work is licensed under a Creative Commons Attribution 4.0 International License. Read Full License 


\section{Abstract}

Object The present study aimed to investigate the methodology and characteristic of the microscopic transsphenoidal resection of pituitary adenomas with a conchal sphenoid sinus.

Method Three patients with sellar tumor and non-pneumatized sphenoid sinuses received microscopic transsphenoidal surgery with help of neuronavigation system.

Result The three conchal spenoid sinuses were accessed safely, total resction was achieved and no serious complication occurred. we found that pituitary tumors with conchal sphenoid sinus was more smaller and soft.

Conclusion the presence of a conchal sphenoid sinus is not an absolute contraindication for employing the microscopic transsphenoidal route in the resction of pituitary adenomas with help of neuronavigation.

\section{Introduction}

The microscopic transsphenoidal route is considered the standard procedure for cure pituitary adenoms, which represent at least $10 \%$ of all intracranial tumors[i]. Transsphenoidal route needs pass through the sphenoid sinus to reach the sella. According to the commonly used classification system proposed by Hammer \& Radberg (1961), the pneumatization of the SS is divided into three types: conchal, presellar, and sellar[ii]. The conchal nonpneumatized sphenoid was always considered to be a contraindication to the transsphenoid approach to the sella. It usually makes this approach less favorable[iii]. The presence of a conchal sphenoid sinus is typically considered to be one of the contraindications for the use of transsphenoidal route due to the difficulties of intraoperative localization and exposure of the sellar floor[iv] $]^{[\mathrm{vl}][\mathrm{V} i]}$. The current study presents three cases of resection of sellar tumors with conchal sphenoid sinus via the microscopic transsphenoidal surgery. Written informed consent was obtained from the patient's family and the patient for all three cases.

\section{Case 1}

\section{History and clinical examination}

35-year-old female, unfortunately falling at home, head hitting the floor, unbearable headache arise after injury, she was been transported urgently to the local hospital, the brain CT scan was performed quickly, found a lesion in the sellar area without intracranial hemorrhage; 4 months later for further examination and treatment she went to our department. Nervous system examination found no obvious signs of neurological deficits. 


\section{Neuroimaging and serum hormones}

MR scan revealed a lesion in the sellar cavity with a concal sphenoid sinus, tumor with height of $1.5 \mathrm{~cm}$, enhanced scan showed a uniform homogeneous enhancement. Serum hormone test found no significant

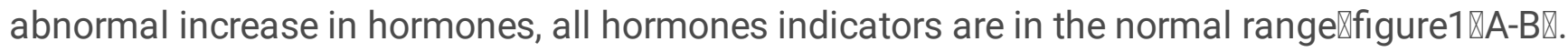

\section{Treatment}

we also applied Microscopic transsphenoidal surgery on this patient with the help of neuronavigation system $\nabla$ figure $1 \mathrm{C}$. The position of patient was supine during procedure of surgery. The incision was in the middle of nasal septum. Using nasal dilator make a surgical corridor between mucosa and bone of septum. It's intermittently employing baton of navigation to find hole of sphenoid sinus accurately, then we gradually drill a tunnel, the diameter was about $1.6 \mathrm{~cm}$, through concal shpenoid sinus to the sellar floor. When the dura of sellar floor was full exposure, we found that dura was lack of blood supply, then the dura was cut off in X-shaped without bleeding of intercavernous sinuses. The tumor tissue that is in solid, intermediate stiffness form and lack blood supply was flowed out through the dura incision. After that the tumor was Gradually removed and carefully protect the normal tissue of pituitary. The CSF

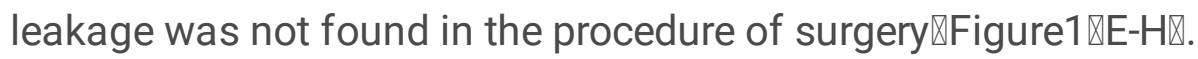

\section{Postoperative course}

After operation the patient recovered quickly, the reexamination of head MRI showed there was no residual tumor 20 months after operation $\otimes$ Figure1D区. Immunohistochemistry: FSH type pituitary adenoma.

\section{Case 2}

\section{History and clinical examination}

25-year-old male patient presented to our hospital's department of Cardiology with chest tightness and asthma for more than 2 months. They consider that this patient may have acromegaly because he has a rough face. The brain MRI examination found the sellar region had a turmor of pituitary, Then he was turned to our department for further treatment. Neurological examination: In addition to special facial changes, the patient also exists oily and thickly skin, sweating, enlargement of heart, heart rate 110 beats/min, lower extremity with moderate edema.

\section{Neuroimaging and serum hormones}

The MR image found that a lesions occupied in the seller region, with height $18 \mathrm{~mm}$, enhanced scaning show that tumor was moderately enhanced; skull bone was unevenly thickening, patched enhancement signal was broadly showed in the diploe of skull bone, the signal is not Uniform. Sphenoid sinus area was full of bone signals, been considered a non- pneumatolytic sphenoid sinus $₫$ Figure $2 \varangle A-B \bigotimes$. Serum GH level was higher than $40 \mathrm{ng} / \mathrm{ml}$, PRL level was equaled $343.99 \mathrm{ng} / \mathrm{ml}$ (reference range 2.1-17.7 ng / ml), IGF- 
1 was $392 \mathrm{ng} / \mathrm{ml}$ (reference range 116-358 ng / ml), Other hormone levels were no obvious abnormalities.

\section{Treatment}

Under the guidance of neuro-navigation, we applied microscopic transsphenoidal surgery to resect the pituitary adenomas. Bilateral nasal of this patient were stenosis and the right stenosis was more significant. Removed the bilateral middle turbinate, left nasal septum before the left side of the incision. Under the guidance of the navigation, and gradually removed the skull base. Finally reached the saddle, exposure of the dura of sella, when the dura was incised by mini knife, we found that the tumor tissue, soft, milky white, blood supplying not rich, flow out from inner sella; as the tumor stopped flow the incision of dura was enlarged by scissors, then the residual tumor was removed totally. No cerebrospinal fluid leakage, there was a little bleeding in cavernous sinus, stopped by packing gelatin sponge on bleeding point. Postoperative pathological immunohistochemistry reported: polyhormonal pituitary adenoma (PRL, GH).

\section{Postoperative course}

At 6 days after surgery, serum GH levels were $12.2 \mathrm{ng} / \mathrm{ml}$, IGF-1: $448 \mathrm{ng} / \mathrm{ml}$ and PRL53.1 ng / ml (reference range 2.1-17.7 ng / ml). Four months after surgery, serum $\mathrm{GH}$ was $7.34 \mathrm{ng} / \mathrm{ml}$. Postoperative MRI examination showed that the lesion of tumor was disappeared $\triangle F i g u r e 2 \triangle C-D \otimes$.

\section{Case 3}

\section{History and clinical examination}

27-year-old female, due to dizziness, progressive obesity, with blurred vision, admitted to our department. she was concentric obesity with full moon face. Binocular vision decreased significantly.

\section{Neuroimaging and serum hormones}

Brain MRI detected an abnormal signal in the right side of the pituitary fossa and found the ossification of sphenoid sinus $\mathbb{F}$ Figure2 $\varangle \mathrm{E}-\mathrm{F} \bigotimes$. T1WI, T2WI showed the lesion with an equal signal, height $7.5 \mathrm{~mm}$, the maximum diameter of $11 \mathrm{~mm}$, dynamic enhanced scan showed some mild enhancement in the area of lesion. Serum hormone test found that morning ACTH 252.4pg / ml (reference range 4.7-48.8 pg / ml), morning serum cortisol 35.65ug / dl (reference range 4.3-22.4ug / dl).

\section{Treatment}

This patient was applied Microscopic transsphenoidal surgery with the guidance of neuronavigation. Using high speed drill to gradually remove the sphenoid bone within the sinus region. when instruments reached the saddle floor that had a thin layer of dense bone we should be take care of that for keeping integrated dura from being drill through. The sellar floor dura was opened, then tumor was easily sucked 
out. We found the tumor was with gray color, soft texture and few blood supply. Finally the tumor was totally removed.

\section{Postoperative course}

One week after surgery, patient's serum ACTH was $14.72 \mathrm{pg} / \mathrm{ml}$ (reference range 4.7-48.8 pg/ ml) and morning serum cortisol was $0.82 \mathrm{ug} / \mathrm{dl}$ (reference range 4.3-22.4ug/dl). Postoperative recovery was smooth, 4.5 months later after surgery the head MRI showed no tumor remnants and recurrence $\triangle F i g u r e 2 \triangle G-H \rrbracket$. Immunohistochemistry conformed that tumor was postive with ACTH.

\section{Discussion}

The presence of a conchal sphenoid sinus is typically considered to be one of the contraindications for the use of transsphenoidal route due to the difficulties of intraoperative localization and exposure of the sellar floor. With advances of the application of microscopy and the application of neural navigation technology, we are able to safely carry out transsphenoidal resection of pituitary adenomas with conchal sphenoid sinus. Compared with the non conchal sphnoid sinus pituitary adenoma, the channel of the transsphenoidal surgery will be smaller, the operation space will be limited, it is more difficult that the surgical instrument be operating in this narrow corridor. We consider that surgical resection of pituitary adenoma with conchal sphnoid sinus under microscope have more advantages than endoscopic transsphnoidal surgery due to microscope surgery need less surgical instruments than endocopic surgery. the distance of the internal carotid artery of the cavernous segment should be carefully measured preoperatively, which determines the size of corridor of TSS surgery[i], which is the adverse aspect to exsect pituitary lesion with conchal sphnoid sinus; during the procedure of drilling conchal sphnoid sinus we should be intermittently apply the probe of neuronavigation to confirm the distance of carotid arteries and to adjust the direction of surgery path to prevent injury the internal carotid arteries accidentally.

But in these four patients of pituitary adenoma with conchal sphnoid sinus, we found, during TSS procedure, that sphenoid boney tissue is relatively loose and easy to drill away, hemostasis of bone surface is not difficult, the sellar floor dura mater is smooth, few vessel, easy to open, Except one case which have very small intercavernous sinus is not caused a significant impact to operation, which may be due to bilateral cavernous sinus blood circulate through a large number of cancellous bone communication instead of the intercavernous sinus of sellar dura, so poor blood supply through sellar dura, bleeding was significantly reduced more rather than those with pneumatized well sphenoid sinus. Loose bone within the sinusoids is easy to drill, bleeding could be stop immediately by the heat and bone meal, when necessary, bone wax can also be used to stop bone bleeding easily. At the same time, we found that the size of pituitary adenoma with conchal sphnoid sinus are smaller, the average diameter is about $1.9 \mathrm{~cm}$, and the tissue of tumor is soft easy removal, and lesions are without rich blood supply, less bleeding. These finds need further research because there are exist same papers already published about that some pituitary adenoma with conchal sphnoid sinus have larger diameter[ii]. Therefore, we believe that pituitary adenoma with conchal sphnoid sinus can relatively safe be resected by transsphenoidal 
surgery with aid of the neuronavigation and achieve satisfactory therapeutic effect, this type PA is no longer a contraindication to transsphenoidal surgery.

To study the anatomy of sphenoid sinus we will find that sphenoid sinus can be divided into conchal type, saddle type, and saddle according to the degree of pneumatolysis; There is study show that the degree of pneumatization of sphenoid sinus play an important role to safely carry out TS surgery, suggest that the circumstance of sphenoid sinus should be full evaluated preoperatively[iii]. Our experience is not only must carry on the MRI scaning preoperatively, also must carry on the thin layer CT scanning of saddle area that would give us more information to comprehensively understand degree of pneumatolysis of sphenoid sinus, and to know the relationship between the internal carotid artery and sphenoid sinus. Conchal type of sphenoid sinus occurrence rate is very low, Song Tao team found that the incidence of sphenoid sinus of nonpneumatized is $6 \%$ in Chinese population[iv], that is consistent with our center study found. Conchal type of pituitary adenoma is a taboo for transsphenoidal surgery in the past, this is due to the thickening of the sphenoid sinus bone cause obstacles to surgery corridor, making the surgeon lost and increasing the risk to injury the internal carotid artery; The application of neuronavigation can better solve this problem, with the aid of neuronavigation improve the success rate of surgery; reduce the rate of complication of operation[v]. In addition, because the bone structure of sphenoid sinus and skull base those structure scanning by MRI is less clear than CT scanning and Studies have shown that error of navigation registration base on CT image is smaller than MRI, we choose CT's neuronavigation, the accuracy is reliable. we are not machinery in accordance with the instruction of the navigation, but we should combin with the experience of surgeons and the observation of sella anatomy, at the same time, to judge the right direction of surgery.

In this study we think pituitary adenomas with conchal sphenoid sinus, the surgery corridor is narrow, so microscopic resection with the aid of neuronavigation has more certain advantages than endoscopic TSS. microscopic surgery use the surgical instruments less than endoscope TTS, so the microscopic TTS are easier to operate, less time needed for surgery; endoscopic observation Angle more wide than microscope[vi], but we found that this type of pituitary adenomas tumors are smaller, no obvious intercavernous sinus, don't need more viewing Angle in resection of pituitary adenoma with conchal sphenoid sinus. although neural endoscopic resection of pituitary adenoma surgery is more and more widely applied, according to our experience, microscopic transsphenoid surgery applying to conchal type of pituitary adenoma has some advantages. at the same time we found that between pituitary adenomas with conchal sphenoid siuns and other type pituitary adenomas exist certain differences, such as anatomical differences, tumor biological behavior characteristics and so on, it remains to be further research.

[i] Yilmazlar S, Kocaeli H, Eyigor O, Hakyemez B, Korfali E. Clinical importance of the basal cavernous sinuses and cavernous carotid arteries relative to the pituitary gland and macroadenomas: quantitative analysis of the complete anatomy. Surg Neurol. 2008;70(2):165-74. 
[ii] Song Y, Wang T, Chen J, Tan G. Endoscopic transsphenoidal resection of sellar tumors with conchal sphenoid sinus: A report of two cases. Oncol Lett, 2015, 9(2):713-716.

[iii] Hamid O, El Fiky L, Hassan O, Kotb A, El Fiky S. Anatomic Variations of the Sphenoid Sinus and Their Impact on Trans-sphenoid Pituitary Surgery. Skull Base, 2008, 18(1):9-15

[iv] Lu Y, Pan J, Qi S, Shi J, Zhang X, Wu K. Pneumatization of the sphenoid sinus in Chinese: the differences from Caucasian and its application in the extended transsphenoidal approach. Journal of anatomy. 2011;219(2):132-42.

[v] Furtado SV, Thakar S, Hegde AS. The use of image guidance in avoiding vascular injury during transsphenoidal access and decompression of recurrent pituitary adenomas. J Craniomaxillofac Surg. 2012;40(8):680-684.

[vi] Zaidi HA, Awad AW, Bohl MA, Chapple K, Knecht L, Jahnke H, et al. Comparison of outcomes between a less experienced surgeon using a fully endoscopic technique and a very experienced surgeon using a microscopic transsphenoidal technique for pituitary adenoma. J Neurosurg. 2016;124(3):596-604.

\section{Conclusion}

the presence of a conchal sphenoid sinus is not an absolute contraindication for employing the microscopic transsphenoidal route in the resction of pituitary adenomas with help of neuronavigation. Pituitary adenomas with conchal sphenoid sinus, the surgery corridor is narrow, microscopic resection with the aid of neuronavigation may has more certain advantages than endoscopic TSS.

\section{Abbreviations}

TSS: Transsphenoidal Surgery; FSH: Follicle-stimulating Hormone囚GH囚Growth hormone; ACTH: Adrenocorticotropic Hormone; CT: Computed Tomography; MRI: Magnetic Resonance Imaging; T1WI: T1weighted Image; T2WI: T2weighted Image

\section{Declarations}

\section{Ethics approval and consent to participate}

The study was conducted in accordance with the Declaration of Helsinki and was approved by the Ethics Committee of 900th Hospital. Written informed consent was obtained from each patient before enrolment in the study.

\section{Consent for publication}

Not applicable 
Availability of data and materials

The datasets used and/or analysed during the current study are available from the corresponding author on reasonable request.

\section{Competing interests}

The authors declare that they have no conflict of interest.

\section{Funding}

None

\section{Authors' contributions}

$\mathrm{HJ}$ collected the patient data and performed the primary writing of the manuscript. SW conceived of this study and participated in its design. All authors read and approved the final manuscript.

\section{Acknowledgements}

Thanks for Doctor Jianjun Gu and Doctor Chenyu Ding' s help and support in this study.

\section{References}

1. Cavallo LM, Messina A, Cappabianca P, et al. Endoscopic endonasal surgery of the midline skull base: anatomical study and clinical considerations. Neurosurg Focus 2005囚19(1): 1-14.

2. Hamberger C A, Hammer G, Marcusson G. Experiences in transantrosphenoidal hypophysectomy. Transactions of the Pacific Coast Oto-Ophthalmological Society annual meeting, 1961, 42(42):27386.

3. Sirikci A, Bayazit YA, Bayram M, Mumbuc S, Gungor K, Kanlikama M. Variations of sphenoid and related structures. Eur Radiol, 2000, 10:844-884.

4. Nomikos $P$, Fahlbusch $R$ and Buchfelder $M$. Recent developments in transsphenoidal surgery of pituitary tumors. Hormones (Athens), 2004, 3: 85-91.

5. Zada G, Agarwalla PK, Mukundan S, Dunn I, Golby AJ, Laws ER. The neurosurgical anatomy of the sphenoid sinus and sellar floor in endoscopic transsphenoidal surgery. J Neurosurg. 2011,114(5):1319-30.

6. Carrabba G, Locatelli M, Mattei L, Guastella C, Mantovani G, Rampini P, et al. Transphenoidal surgery in acromegalic patients: anatomical considerations and potential pitfalls. Acta Neurochir (Wien). 2013;155(1):125-30.

7. Yilmazlar S, Kocaeli H, Eyigor O, Hakyemez B, Korfali E. Clinical importance of the basal cavernous sinuses and cavernous carotid arteries relative to the pituitary gland and macroadenomas: quantitative analysis of the complete anatomy. Surg Neurol. 2008;70(2):165-74. 
8. Song $Y$, Wang T, Chen J, Tan G. Endoscopic transsphenoidal resection of sellar tumors with conchal sphenoid sinus: A report of two cases. Oncol Lett, 2015, 9(2):713-716.

9. Hamid O, El Fiky L, Hassan O, Kotb A, El Fiky S. Anatomic Variations of the Sphenoid Sinus and Their Impact on Trans-sphenoid Pituitary Surgery. Skull Base, 2008, 18(1):9-15

10. Lu Y, Pan J, Qi S, Shi J, Zhang X, Wu K. Pneumatization of the sphenoid sinus in Chinese: the differences from Caucasian and its application in the extended transsphenoidal approach. Journal of anatomy. 2011;219(2):132-42.

11. Furtado SV, Thakar S, Hegde AS. The use of image guidance in avoiding vascular injury during transsphenoidal access and decompression of recurrent pituitary adenomas. J Craniomaxillofac Surg. 2012;40(8):680-684.

12. Zaidi HA, Awad AW, Bohl MA, Chapple K, Knecht L, Jahnke H, et al. Comparison of outcomes between a less experienced surgeon using a fully endoscopic technique and a very experienced surgeon using a microscopic transsphenoidal technique for pituitary adenoma. J Neurosurg. 2016;124(3):596-604.

\section{Figures}

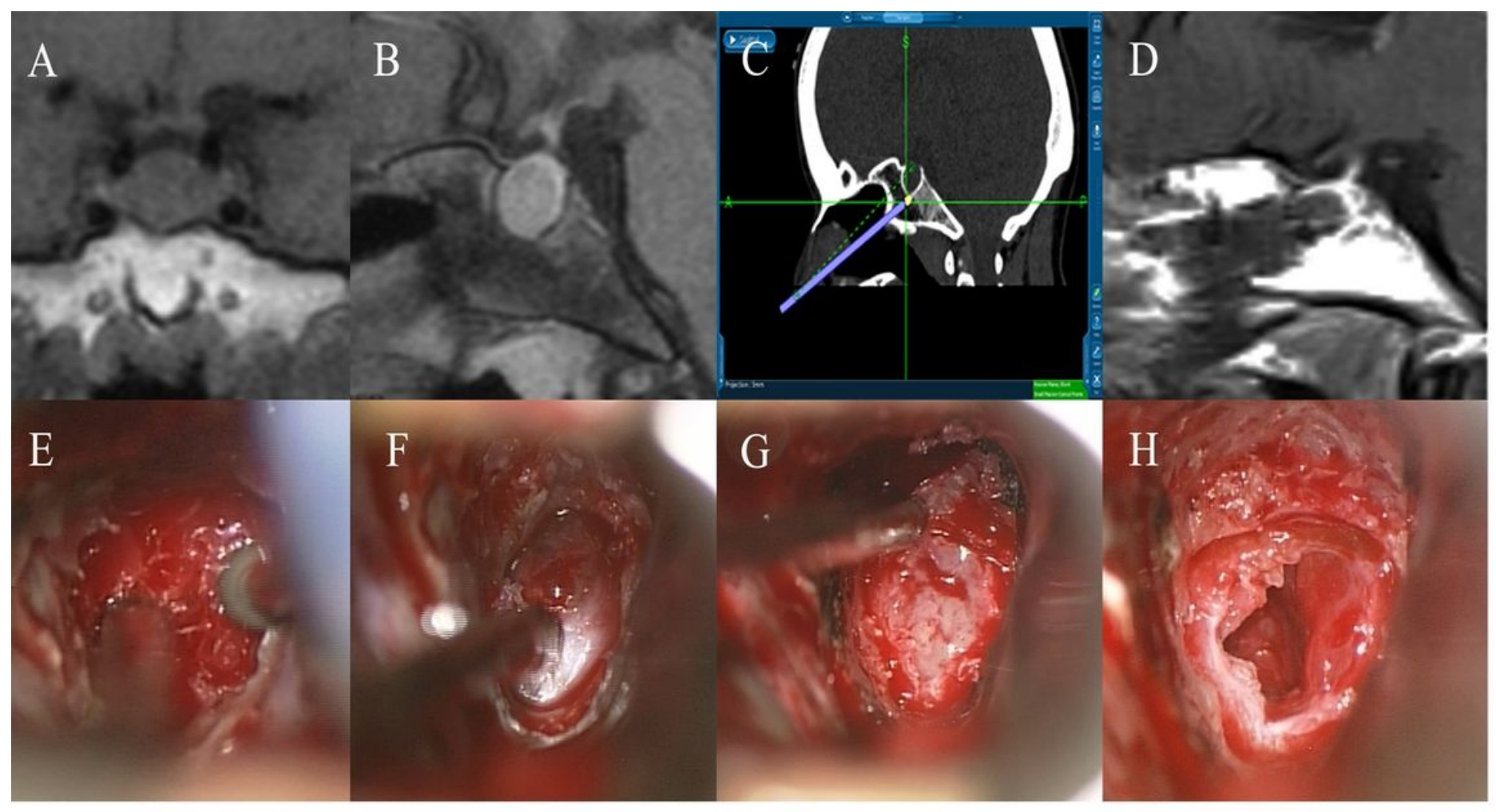

\section{Figure 1}

The preoperative MR imaging and intraoperative picture of Case one: $A \sim B$, Preoperative MR image showed that saddle area occupied by the pituitary adenomas with concal sphenoid sinus. C, Neuronavigation guided the surgical direction. D, Postoperative MR images revealed complete tumor 
resection. $E$, Open the anterior wall of sphenoid sinus after exposure of ossification of the sphenoid sinus. F, Open the sellar floor display dural thick, no obvious intercavernous sinus. G, Soft tumor texture, less blood supply. $\mathrm{H}$, The medial wall of cavernous sinus was revealed after tumor resection.

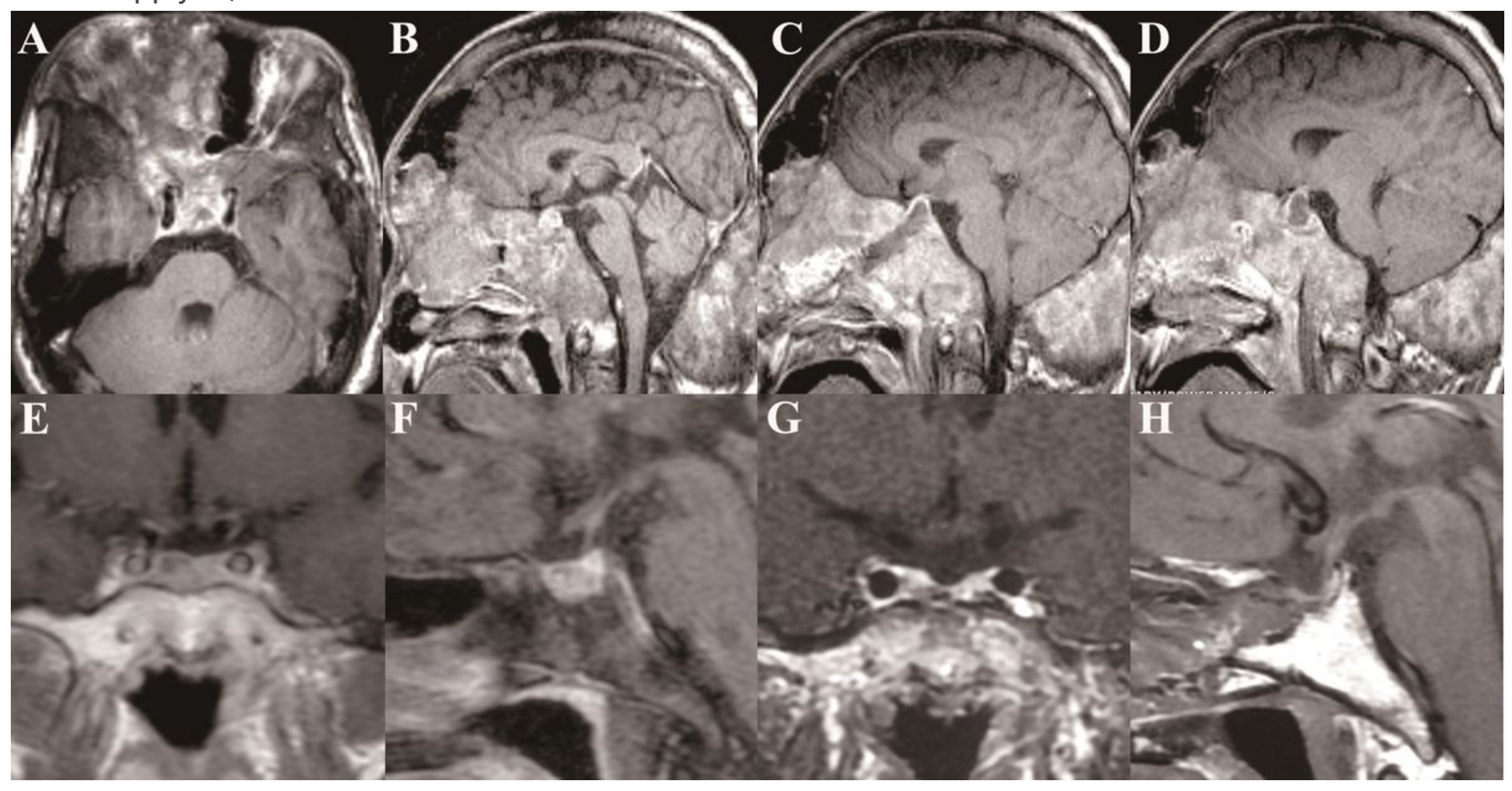

Figure 2

Preoperative and postoperative MR imaging of Case two and Case three: A-B: case 2 preoperative axial and sagittal magnetic resonance imaging, found a lesion in the saddle area, the maximum diameter of tumor was $18 \mathrm{~mm}$, sphenoid sinus calcification, and visible skull fibrous hyperplasia. C-D: Case 2 postoperative 2 months review of magnetic resonance imaging, there had a bony surgical pathway in the sphenoid sinus, no tumor recurrence. E-F: case 3 preoperative coronal and sagittal magnetic resonance imaging, found a microadenomas on the right side of the pituitary fossa, with conchal sphenoid sinus. G$\mathrm{H}$ : Case 3 postoperative MR image after surgery of 4.5 months showed no tumor recurrence. 